\title{
Pressure and temperature dependence of the laser-induced plasma plume dynamics
}

\author{
Alejandro Ojeda-G-P, ${ }^{1}$ Christof W. Schneider, ${ }^{1}$ Thomas Lippert, ${ }^{1,2}$ and Alexander Wokaun ${ }^{1}$ \\ ${ }^{1}$ Energy and Environment Department, Paul Scherrer Institut, 5232 Villigen-PSI, Switzerland \\ ${ }^{2}$ Laboratory of Inorganic Chemistry, Department of Chemistry and Applied Biosciences, ETH Zürich, \\ CH-8093 Zürich, Switzerland
}

(Received 3 July 2016; accepted 17 November 2016; published online 8 December 2016)

\begin{abstract}
The influence of different background gases and substrate heating on the plasma plume dynamics from silver ablation is investigated by species selected time and space resolved imaging. The results provide a time-resolved understanding on how those process parameters affect the expansion: from a free expansion in vacuum with velocities exceeding $20000 \mathrm{~m} / \mathrm{s}$ to a very slow expansion in Ar at $1 \times 10^{-1}$ mbar with arrival velocities of $280 \mathrm{~m} / \mathrm{s}$. In addition, we observe a rebound of the ablated material on the substrate holder leading to a re-coating of the ablated target. At $1 \times 10^{-1}$ mbar, it seems that the expansion of the plasma plume displaces a considerable portion of the background gas and traps it against the frontal area of the substrate holder. This leads to a transient high local pressure just above the substrate. In the case of Ar, the rebound is enhanced due to inelastic scattering, whereas for an $\mathrm{O}_{2}$ background, an area of high reactivity/emission in addition to the rebound is created. Imaging of selected species shows that the light emission in this area is mainly due to excited Ag and metal oxygen species. There is a clear influence of substrate heating on the plasma expansion due to the background gas density gradients, reducing the stopping ability of the background gas and already detectable $2 \mathrm{~cm}$ away from the substrate. Both rebound and excitation effects are reduced in intensity due to the substrate heating. Published by AIP Publishing.

[http://dx.doi.org/10.1063/1.4971251]
\end{abstract}

\section{INTRODUCTION}

Pulsed laser deposition (PLD) is one of the preferred methods for investigating and preparing thin films for a wide range of materials and applications. It is a very versatile technique that gained its status after discovering its capability to transfer the composition of complex target materials to thin films. ${ }^{1-5}$ Despite its simple operating principle (ablating a target material with a laser pulse and depositing the ejected material on a substrate), the complexity of the process is remarkable. The effects of most of the process parameters have been studied in detail, and their basic understanding can be found in numerous publications in, e.g., Refs. 2, 3, and 6 and references therein.

Time resolved imaging is a diagnostic technique that can provide a deeper insight into the PLD process. It provides a spatially resolved understanding of the plume expansion dynamics and how the different process parameters are affected. One of the most common parameters studied using imaging has been the background pressure and the type of gas used, ${ }^{7-9}$ like the detection of atomic oxygen and metal oxygen species in the near substrate region when ablating YSZ (yttria stabilized zirconia) in $\mathrm{O}_{2}{ }^{7}$ or a $3 \mathrm{~mm}$ luminescent rebound above the substrate which is attributed to collisions of the plume with species reflected from the substrate. ${ }^{8}$

Additional studies have analysed the influence of substrate heating on the plasma plume expansion. ${ }^{10-12}$ This is a parameter which is considered to have a strong influence on the film growth once the species arrive at the substrate, but does not seem to have a direct influence on the plume expansion. However, those studies show that it does have an influence, as the heat transfer from the substrate to the background gas creates a density gradient that affects the expansion dynamics of the plasma plume. This influence of substrate temperature on the background gas density and deposition rates has been modelled mathematically in Refs. 10 and 11 using a simplified model of plume front propagation from Ref. 13 which resolves the equation of motion between the traveling mass of the plume in addition to the swept gas and the external pressure force. The changes in the background gas density are accounted by assuming conductive heat transfer and a temperature jump at the walls and calculating the position resolved density of the background gas.

In this study, we investigate by time-resolved imaging the influence of different background gases, pressures, and substrate heating on the plasma plume expansion by ablating silver as a model system. It continues the investigation performed by Amoruso et al. ${ }^{10-12}$ and encounters plasma plume rebounds, target re-coating, and locally enhanced transient pressures, something which directly affects the film growth and the composition.

\section{EXPERIMENTAL DETAILS}

The ablation experiments were performed using a $\mathrm{KrF}$ excimer laser (Lambda Physik LPX 300, 20 ns pulses, $\lambda=248 \mathrm{~nm}$ ). A mask was used to obtain a flat-top beam profile and to image a rectangular laser spot with dimensions of $1 \mathrm{~mm} \times 1.4 \mathrm{~mm}$ on a cylindrical silver target. The experiments were carried out with a fluence of $3 \mathrm{~J} / \mathrm{cm}^{2}$ at a repetition rate of $4 \mathrm{~Hz}$. The target was continuously displaced and 
rotated to avoid cratering, and the target-to-substrate distance was set at $40 \mathrm{~mm}$.

Two different background gases were used: Ar and $\mathrm{O}_{2}$. The former would give an insight into the collisional interactions with limited chemical reactions, whereas the latter combines both and is a typical gas used for PLD. The experiments were performed in vacuum and at pressures of $1 \times 10^{-2} \mathrm{mbar}$ and $1 \times 10^{-1}$ mbar (related to the mean free path, MFP). The pressures were chosen to capture the evolution from a free expansion of the plasma plume (vacuum), to slight interactions with the background gas (MFP of $\sim 20 \mathrm{~mm}$ at $1 \times 10^{-2} \mathrm{mbar}$ ) and to a full interaction (MFP of $\sim 2 \mathrm{~mm}$ at $1 \times 10^{-1} \mathrm{mbar}$ ). They were performed at room temperature, $600^{\circ} \mathrm{C}$, and in some cases at $800^{\circ} \mathrm{C}\left(\mathrm{O}_{2}\right)$ to analyse the influence of the substrate heating on the plasma expansion dynamics.

It is noteworthy that the indicated pressures of $1 \times 10^{-2}$ mbar and $1 \times 10^{-1}$ mbar for Ar (measured using a Pfeiffer Vacuum PBR 260 Compact full range sensor: hot cathode and Pirani sensor) correspond to effective pressure values of 1.6 $\times 10^{-2}$ mbar and $1.6 \times 10^{-1}$ mbar, respectively. These small differences have no influence on the data when the differences in pressure are of orders of magnitude and for clarity the indicated pressures for Ar were kept. In the case of $\mathrm{O}_{2}$ background gas, the indicated and effective pressures are the same.

An intensified charge-coupled device (ICCD) "Andor New i-star" was used to record the time evolution of the plasma plume. The sensor has a $1024 \times 1024$ pixels size, and the pixel to distance ratio was calculated using the reference target-to-substrate distance. The images were recorded for either all light passing through a quartz window $(200 \mathrm{~nm}-$ $1000 \mathrm{~nm}$ ) or for selected wavelengths using an acousto-optic tunable filter (AOTF, 550-1000 nm). The AOTF (Brimrose models VA210-0.55-1.0-H) has a wavelength resolution of $0.6-3 \mathrm{~nm}$ with a manufacturer certified resolution of $1.3 \mathrm{~nm}$ at $633 \mathrm{~nm}$ and $2 \mathrm{~nm}$ at $800 \mathrm{~nm}$.

An image of the experimental setup can be seen in Fig. 1. To record the images, the camera was triggered externally

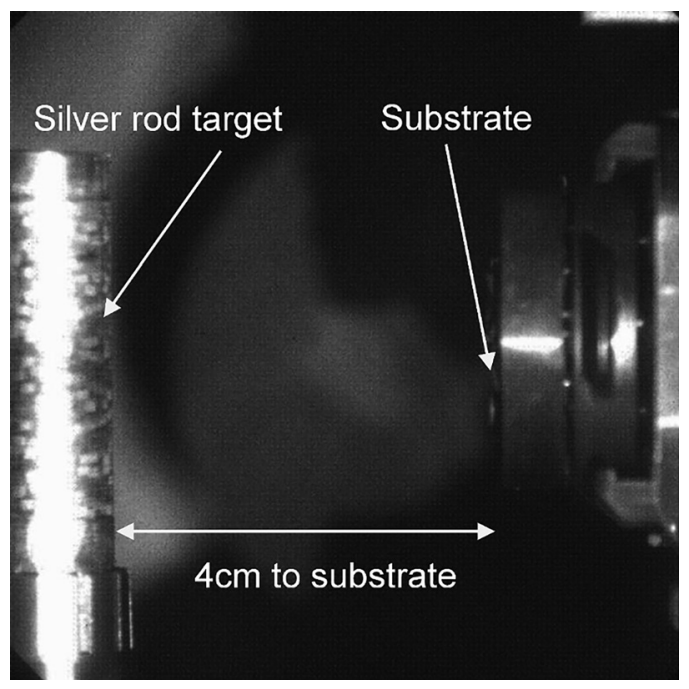

FIG. 1. Image of the experimental setup with a silver rod target on the left and a substrate $4 \mathrm{~cm}$ away on the right hand side. The substrate is mounted on a heated substrate-holder. The image was captured using the same ICCD as for the experiments. by the laser pulse using a photo-diode. An on-board digital delay generator was used for gating the image intensifier (typically with a gate width of $50 \mathrm{~ns}$ ), and increasing timedelays were used to capture its time evolution. For each time-frame, 40 images were accumulated to improve the signal to noise ratio.

Plasma chemical interactions were investigated by spectroscopic analyses using a monochromator (Acton research Spectra Pro 500). The light emitted by the plasma plume between the target and the substrate was captured using an achromatic lens $(200-2000 \mathrm{~nm}, f=500 \mathrm{~mm}$ and $50 \mathrm{~mm}$ diameter) directly in front of the window, and the beam was then directed to the monochromator using a set of mirrors. The emission spectra were recorded for vacuum, Ar, and $\mathrm{O}_{2}$ background gases (both at $1 \times 10^{-1}$ mbar). Initially, a coarse grating was used to capture a broader spectrum from 500 to $830 \mathrm{~nm}$ (Fig. 2(a)), whereas a higher resolution measurement was subsequently performed between 760 and $830 \mathrm{~nm}$ (Fig. 2(b)). In this case, the region of interest was divided into smaller range measurements $(\sim 15 \mathrm{~nm}$, using a high resolution grating), which were then assembled together. These measurements were triggered by the same photodiode mentioned above and gated using a Princeton Instruments pulse

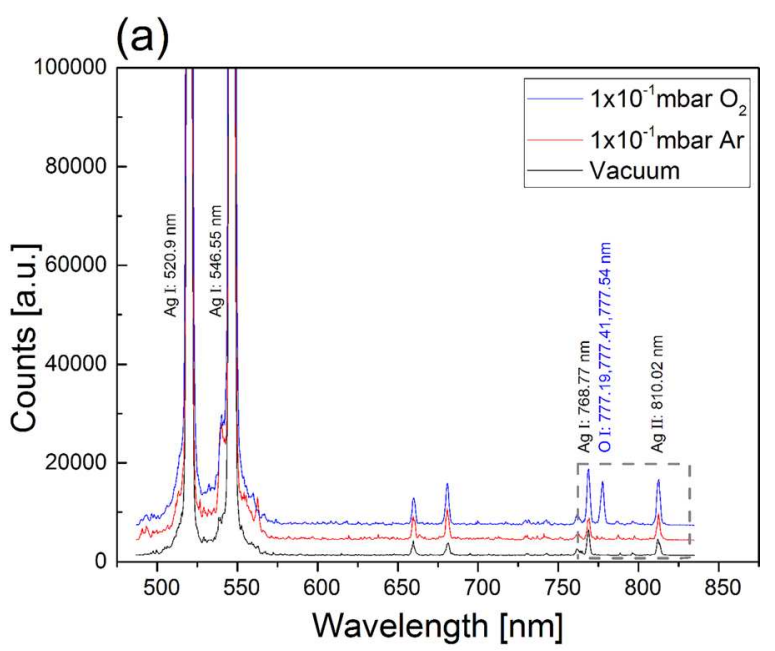

(b)

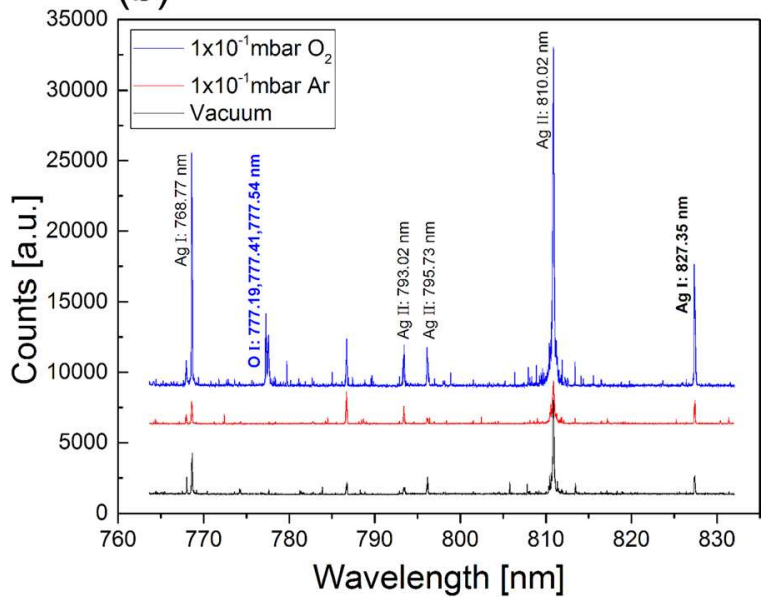

FIG. 2. Emission spectra for silver ablation in vacuum, $\mathrm{Ar}$ and $\mathrm{O}_{2}$ (both at $1 \times 10^{-1}$ mbar) for wavelengths of $500-830 \mathrm{~nm}$ (a) and with a higher resolution for $765-830 \mathrm{~nm}$ (b). 
generator (PG-200). An accumulation of 20 measurements per image was selected to improve the signal to noise ratio with a delay of $200 \mathrm{~ns}$ and a gating width of $10 \mu \mathrm{s}$.

The emission spectra from the silver ablation show a low number of emissions lines compared to other elements from the periodic table and are characteristically green to the human eye as the high intensity emission lines at 520.9 and $546.55 \mathrm{~nm}$ confirm (Fig. 2(a)). There is a clear quenching of the emission intensity in $\mathrm{Ar}$ in the $765-830 \mathrm{~nm}$ range (Fig. 2(b)), whereas in $\mathrm{O}_{2}$, several overlapping high intensity peaks of O I arise $(777.19,777.41$, and $777.54 \mathrm{~m})$ and most emission intensities increase (higher number of excitations due to chemical reactions with $\mathrm{O}_{2}$ ).

The emission lines were identified using References 14 and 15, and the selected ones are listed in Table I. The selection was based on emission lines being among the strongest for the particular species, attainable by the range of the AOTF (550-1000 nm) and sufficiently separated to allow correct imaging, although for Ar the selection was based on the recommended lines from Ref. 15. The selected wavelength values were from neutral (I) rather than ionized states (II) and were then used for the AOTF measurements to allow the time-resolved imaging of the different species.

\section{RESULTS AND DISCUSSION}

\section{A. Room temperature analyses}

The measurements performed without substrate heating are shown in Fig. 3 for the different pressure conditions and background gases. All images are taken with the same aperture and exposure times. The intensity scales are identical for all images to provide a clear understanding of the time evolution of emission intensities, even if this causes out-ofrange values below $5 \mu \mathrm{s}$.

The plasma plume expansion in vacuum is the fastest to reach the substrate in less than $2 \mu$ s and shows a strong forward peaked shape. Interestingly, after reaching the substrate, there is a noticeable rebound of plasma species that modify the shape of the plasma front and push it towards the ablated target (visible at $5 \mu$ s and $7 \mu$ s images). With an increase of pressure to $1 \times 10^{-2} \mathrm{mbar}$, the arrival is delayed for both background gases, $\mathrm{O}_{2}$ and $\mathrm{Ar}\left(t_{O_{2}} \approx 2.75 \mu \mathrm{s}\right.$ and $\left.t_{A r} \approx 3 \mu \mathrm{s}\right)$. At such pressure, the MFP becomes shorter than the target-to-substrate distance and collisions with the background gas are expected. The difference in expansion vs. vacuum is already visible at $1 \mu$ s with vacuum presenting a

TABLE I. Used emission wavelengths of Ag, O, and Ar by either arc, spark, or discharge excitation. ${ }^{14,15}$ Remark: intensities are rough indicators as different observers use different scales and excitation sources.

\begin{tabular}{lccc}
\hline \hline Species & State & Emission wavelength (nm) & Intensity \\
\hline $\mathrm{O}$ & $\mathrm{I}$ & 777.19 & 870 \\
$\mathrm{O}$ & $\mathrm{I}$ & 777.41 & 810 \\
$\mathrm{O}$ & $\mathrm{I}$ & 777.54 & 750 \\
$\mathrm{Ar}$ & $\mathrm{I}$ & 811.53 & 35000 \\
$\mathrm{Ag}$ & $\mathrm{I}$ & 827.35 & 290 \\
\hline \hline
\end{tabular}

more elongated profile. The rebound effect is now hardly visible. Comparing $\mathrm{O}_{2}$ and $\mathrm{Ar}$ as background gases, the latter shows a later time of arrival due to its higher stopping ability (already visible at $3 \mu \mathrm{s}$ ), a consequence of the different masses $(\mathrm{A}=32$ vs. $\mathrm{A}=40)$ and also the van der Waals volume. The molecular volume of $\mathrm{O}_{2}$ is $23.013 \AA^{3}$ (calculated using the van der Waals radius of $1.52 \AA$ and an interatomic distance of $1.2074 \AA$ (Refs. 14 and 16)), whereas the atomic volume of $\mathrm{Ar}$ is $27.83 \AA^{3}$ (van der Waals radius of $1.88 \AA$ (Ref. 14)).

At $1 \times 10^{-1}$ mbar, the arrival times for $\mathrm{O}_{2}$ and Ar are ${ }_{t O} O_{2} \approx 9.75 \mu$ s and $t_{A r} \approx 28 \mu \mathrm{s}, \sim 5-10$ times longer than in vacuum. The arrival times have been estimated by monitoring the first signal of arrival of light emitting species at the substrate. Apart from the arrival times, there are also changes in the plasma plume shape and light emission. In both cases, the plasma plume quickly transforms from a forward peaked to a broadened spherical geometry (in the $2-5 \mu$ s range), which, although we report $2 \mathrm{D}$ images of the expansion, is a 3D shape and resembles the expansion of an air bubble in water.

In terms of emission, there is a considerable amount of excitations evidenced by the longer emission times. This takes place mostly at the plasma plume front for $\mathrm{Ar}$ and around its entire perimeter for $\mathrm{O}_{2}$ (a clear example can be seen in Fig. 3 at $t=7 \mu$ s and marked with an inclined arrow). In the latter, it highlights chemical reactions between the plasma plume outer species and the $\mathrm{O}_{2}$ background to form metal oxygen species and is further analysed in Section III A 1. For Ar, it is mostly excitations due to collisional interactions taking place at the centre of the plasma front where the species travel with the highest energies.

On a larger time scale $(>13 \mu \mathrm{s}), \mathrm{O}_{2}$ shows an intense excitation of species just above the substrate (marked with an arrow in Fig. 3 at $t=17 \mu \mathrm{s}$ ). It covers the substrate and its holder, resembling a boundary layer of $5 \mathrm{~mm}$ thickness, and lasts for $\sim 20 \mu$ s, twice the time it took the plasma to arrive at the substrate. A time resolved intensity profile is shown in Fig. 4(a) to highlight the effect. It shows that once the plasma arrives at the substrate $(\sim 10 \mu \mathrm{s})$, there is a considerable increase in light emission, which is even higher than the intensities seen at $5 \mu$ s and lasts up to $27 \mu$ s before gradually disappearing (Fig. 3 at $33-51 \mu$ s).

We believe that this is a consequence of the trapping of the $\mathrm{O}_{2}$ background against the frontal area of the holder as it is displaced by the expansion of the plasma plume. Hence, an $\mathrm{O}_{2}$ rich volume is squeezed just on top of the substrate/ holder creating a transient high local $\mathrm{O}_{2}$ pressure, leading to a very reactive environment. This means that most of the deposited species will mainly react chemically with the $\mathrm{O}_{2}$ gas in the last $5 \mathrm{~mm}$ of their path, instants before arriving to the substrate.

This locally enhanced pressure is, as far as we are aware of, not implemented in any PLD mathematical model developed to date and which would have a critical role on deposition rates, chemistry, and dynamics. ${ }^{11,17,18}$ An approximation of the value of this transient pressure can be obtained by using Boyle's gas law ( $P V=$ constant). If it is assumed that the temperature and the amount of gas remains unchanged, a 


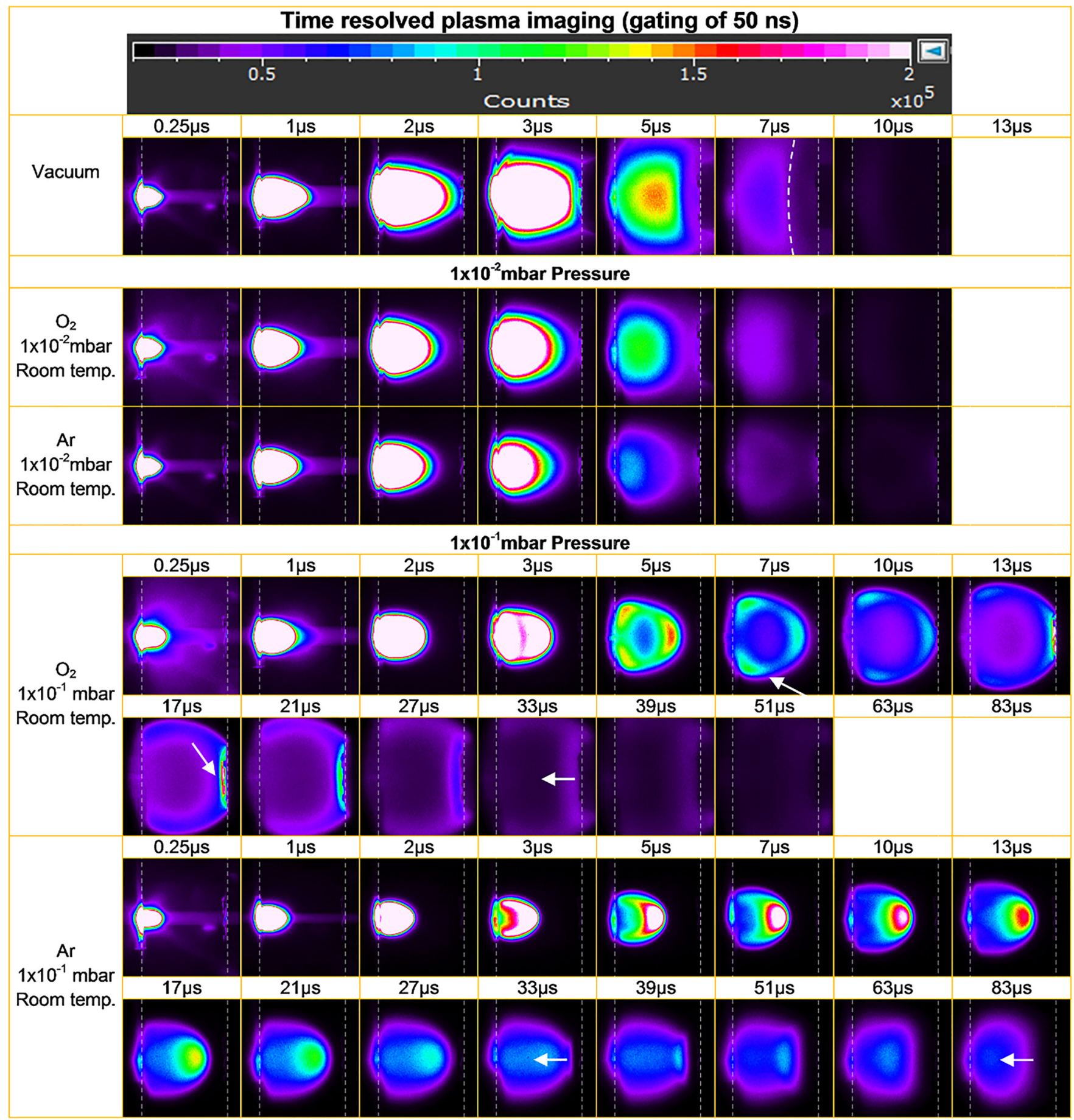

FIG. 3. ICCD time resolved plasma imaging for vacuum, $1 \times 10^{-2} \mathrm{mbar}$ and $1 \times 10^{-1} \mathrm{mbar}$ for $\mathrm{O}_{2}$ and Ar background gases without substrate heating. Images of visible light $(200 \mathrm{~nm}-1000 \mathrm{~nm})$ with no filter applied. The plume expands from the ablated target on the left to the substrate on the right (both marked with vertical dashed lines).

piston like compression of a cylindrical volume $(\varnothing$ of the substrate holder) from a length of $30 \mathrm{~mm}$ to one of $3 \mathrm{~mm}$ is equivalent to a 10 fold rise in pressure. This would correspond to a local transient pressure of 1 mbar vs. the original 0.1 mbar of the background gas.

With respect to the Ar measurements at $1 \times 10^{-1} \mathrm{mbar}$ (Figs. 4(b) and 5(b)), there is no excitation/light emitting volume on top of the substrate holder. Just $5 \mathrm{~mm}$ before reaching the substrate, the light emitting plasma plume is stopped, no light is emitted from this region above the substrate (marked with an arrow in Fig. 5(b)) and instants later, a rebound wave can be seen traveling backwards (visible in the dashed intensity profiles in Fig. 4(b)). This is also visible in Fig. 3, starting at circa $31 \mu$ s and progressing up to $83 \mu \mathrm{s}$ when some rebounded species arrive to the ablated rod target. This rebound follows a different mechanism to those seen in vacuum and $1 \times 10^{-2}$ mbar conditions, as it is not caused by the species reflected after hitting the substrate.

As with $\mathrm{O}_{2}$, it seems that the rapid expansion of the plasma plume compresses a portion of Ar gas against the 

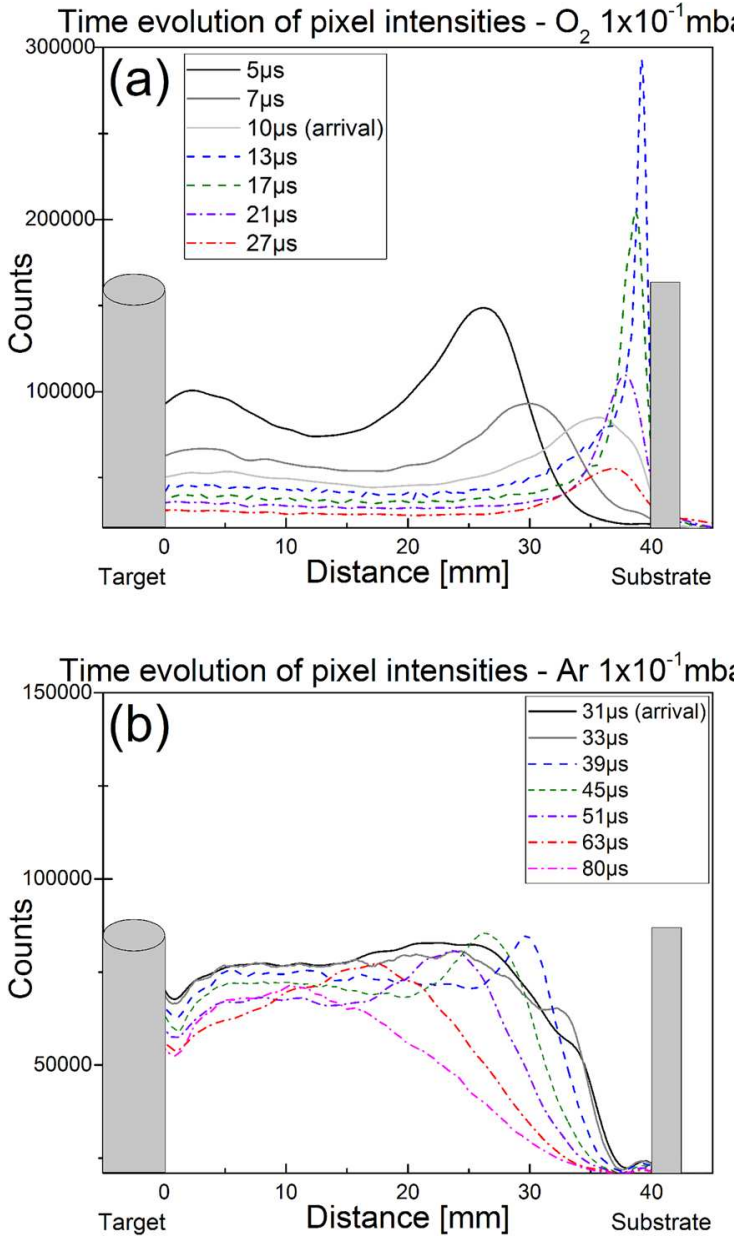

FIG. 4. Time-resolved intensity profiles along the centre of the plasma plume from the ablated target to the substrate, for a background gas pressure of $1 \times 10^{-1} \mathrm{mbar} \mathrm{O}_{2}$ (a) and $\mathrm{Ar}(\mathrm{b})$, both at room temperature.

substrate holder. This compressed gas acts then as a spring energy storage, and once the plasma plume is stopped, it accelerates it towards the target due to the pressure gradient (between the high local pressure and the vacuum left after the plume expanded and swept away the background gas). Surprisingly, this rebound travels at a higher velocity than the one of arrival to the substrate $(\sim 530 \mathrm{~m} / \mathrm{s} \pm 40 \mathrm{~m} / \mathrm{s}$ vs. the arrival of $\sim 280 \mathrm{~m} / \mathrm{s} \pm 47 \mathrm{~m} / \mathrm{s}$ ). A velocity which is nearly constant during the return to the target (measured from $t=35-57 \mu \mathrm{s})$ as it encounters no collisions with gas species to slow it down.

The rebound arrives at the ablated target $\sim 100 \mu \mathrm{s}$ after the laser shot, thus redepositing material on its surface and very likely affecting its surface composition if it would be a multi-element target.

\section{Time and space resolved study of selected species}

The intense light emission close to the substrate surface observed in Fig. 5(b) was subsequently analysed by recording the O I and Ag I emission wavelengths (Fig. 6). No significant $\mathrm{O} I$ excitation was detected at that location, whereas Ag I showed a noticeable enhancement. Although it does not seem to be the sole contributor to it as the characteristic
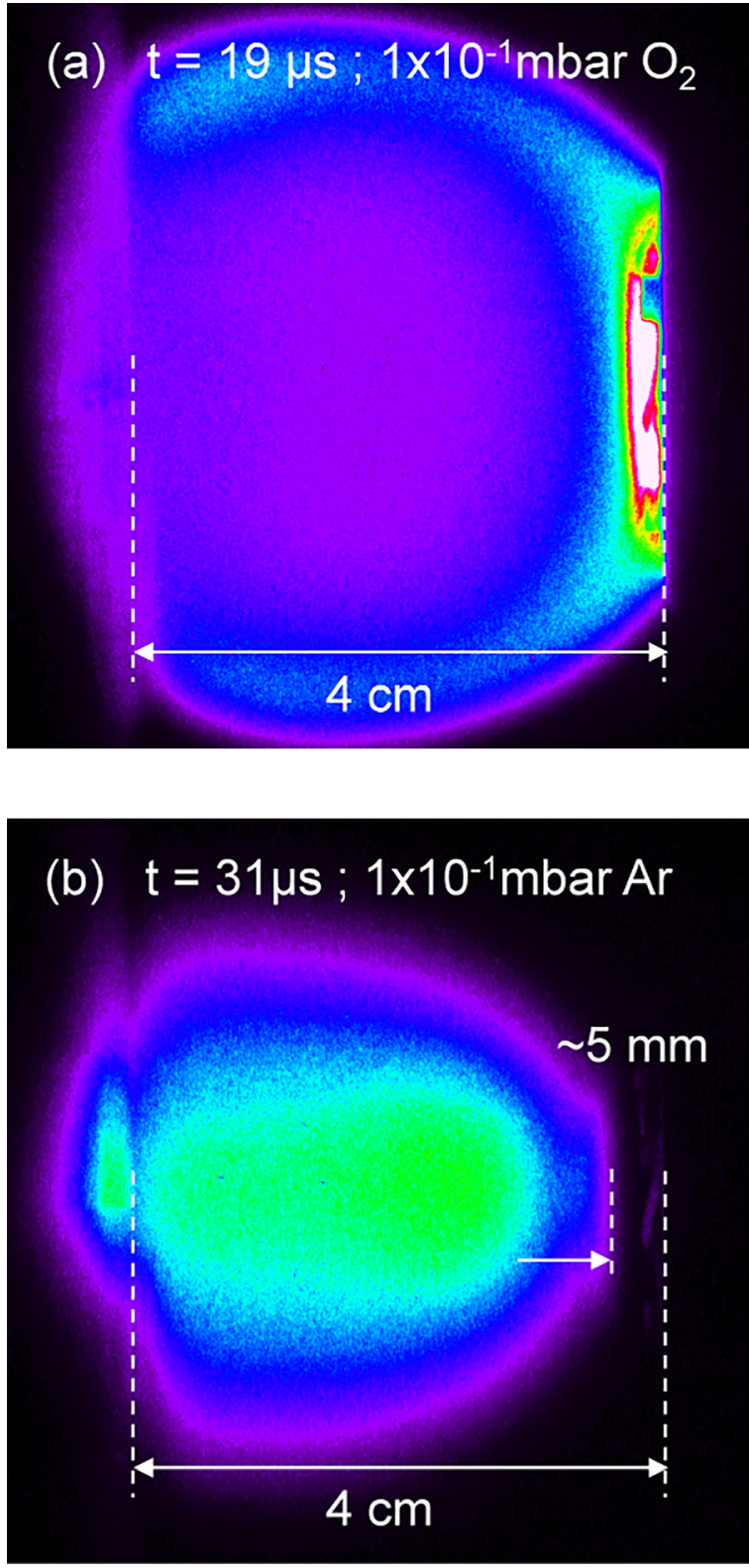

FIG. 5. Plasma plume arrival behaviour for $1 \times 10^{-1} \mathrm{mbar}_{2}$ (a) (for $t=19 \mu \mathrm{s}$ ) and $\operatorname{Ar}(\mathrm{b})$ (for $t=31 \mu \mathrm{s}$ ), both at room temperature. The nonemitting area close to the substrate is highlighted in (a) as well as the positions of the target and the substrate. Both images share the same intensity scale.

emission in the perimeter of the plume does not come from neither $\mathrm{Ag}$ I nor O I.

No AgO I emission lines could be found in the literature and no information concerning the temporal and spatial distributions of this species could be recorded. Due to this deficiency of our model system (Ag), we chose to repeat the experiment with $\mathrm{La}_{0.4} \mathrm{Ca}_{0.6} \mathrm{MnO}_{3}$, as for this particular material, LaO I emission lines are well known. ${ }^{14}$ The emission line of $560.25 \mathrm{~nm}$ was chosen, and the results can be seen in Fig. 7 together with those of other species.

For $\mathrm{La}_{0.4} \mathrm{Ca}_{0.6} \mathrm{MnO}_{3}$, the arrival time to the substrate is shorter than for $\mathrm{Ag}$ for the same $\mathrm{O}_{2}$ gas pressure $\left(\mathrm{La}_{0.4} \mathrm{Ca}_{0.6} \mathrm{MnO}_{3}\right.$ presents higher ablation rates which result in a more efficient displacement of the background gas). This is seen for the general plasma emission image Fig. 7(b), 

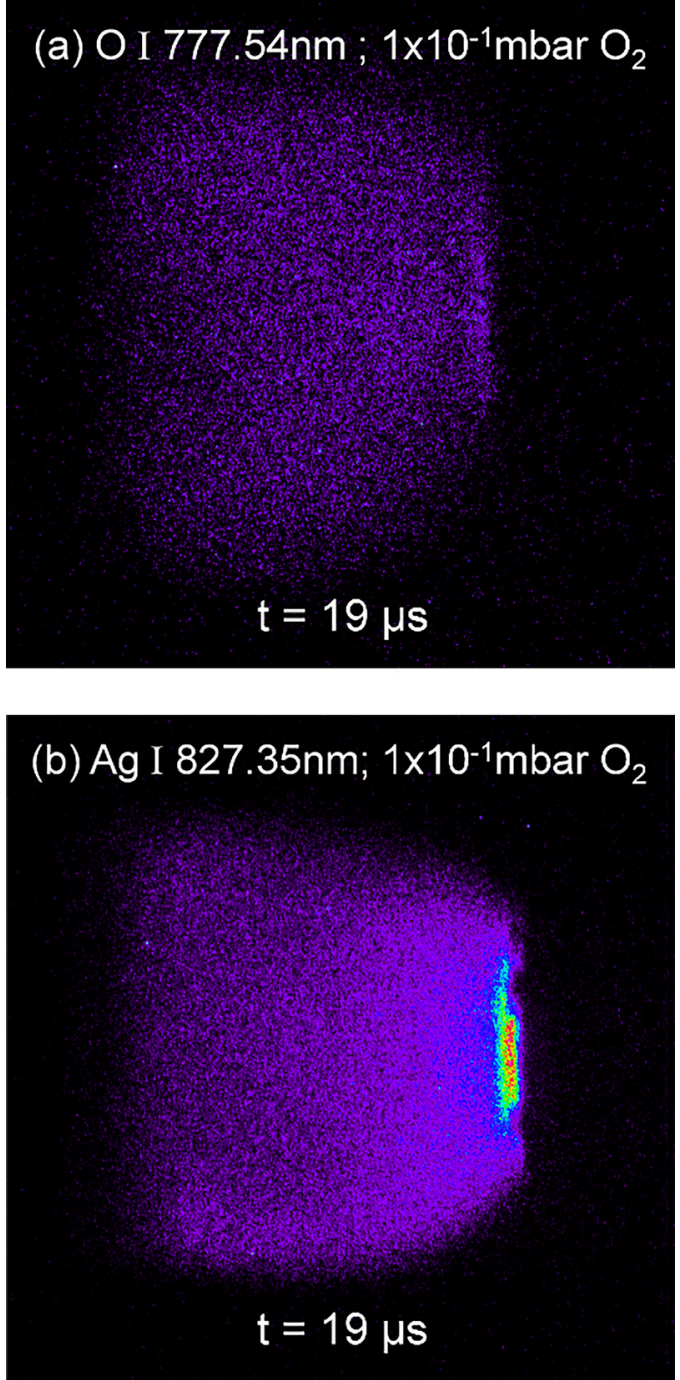

FIG. 6. ICCD images showing the spatial distributions at $t=19 \mu$ s of O I (a) and $\mathrm{Ag} \mathrm{I}(\mathrm{b})$. An AOTF filter was used to capture the emission wave-lengths of $777.54 \mathrm{~nm}$ (for O I) and $827.35 \mathrm{~nm}$ (for Ag I). Due to the reduced light transmission when using the AOTF, a gating time of $1 \mu \mathrm{s}$ and 100 accumulations were necessary to ensure visibility. The emission scales were selected to ensure the maximum contrast.

as well as for the different spatial distributions of species. With the spatial distribution of LaO I showing the high emissivity area close to the substrate Fig. 7(d), proving that this area with high oxygen concentration favours the generation of metal oxygen species. Additionally, at this specific timeframe of $16 \mu \mathrm{s}$, the late arrival of O I (the lightest participating element and easier to stop) is captured while it is still approaching the substrate (Fig. 7(f)).

\section{Analysis of position-resolved plasma velocities}

To provide a better understanding of the expansion dynamics for the different pressures, a position-resolved velocity chart is presented in Fig. 8. The chart shows the traveling velocities of the plasma front calculated by averaging the distances travelled with respect to time of 3 consecutive frames ( $250 \mathrm{~ns}$ delay between frames).

They reveal initial velocities of $\sim 28000 \mathrm{~m} / \mathrm{s}$ which for the case of vacuum remain constant at $\sim 20000 \mathrm{~m} / \mathrm{s}$ during
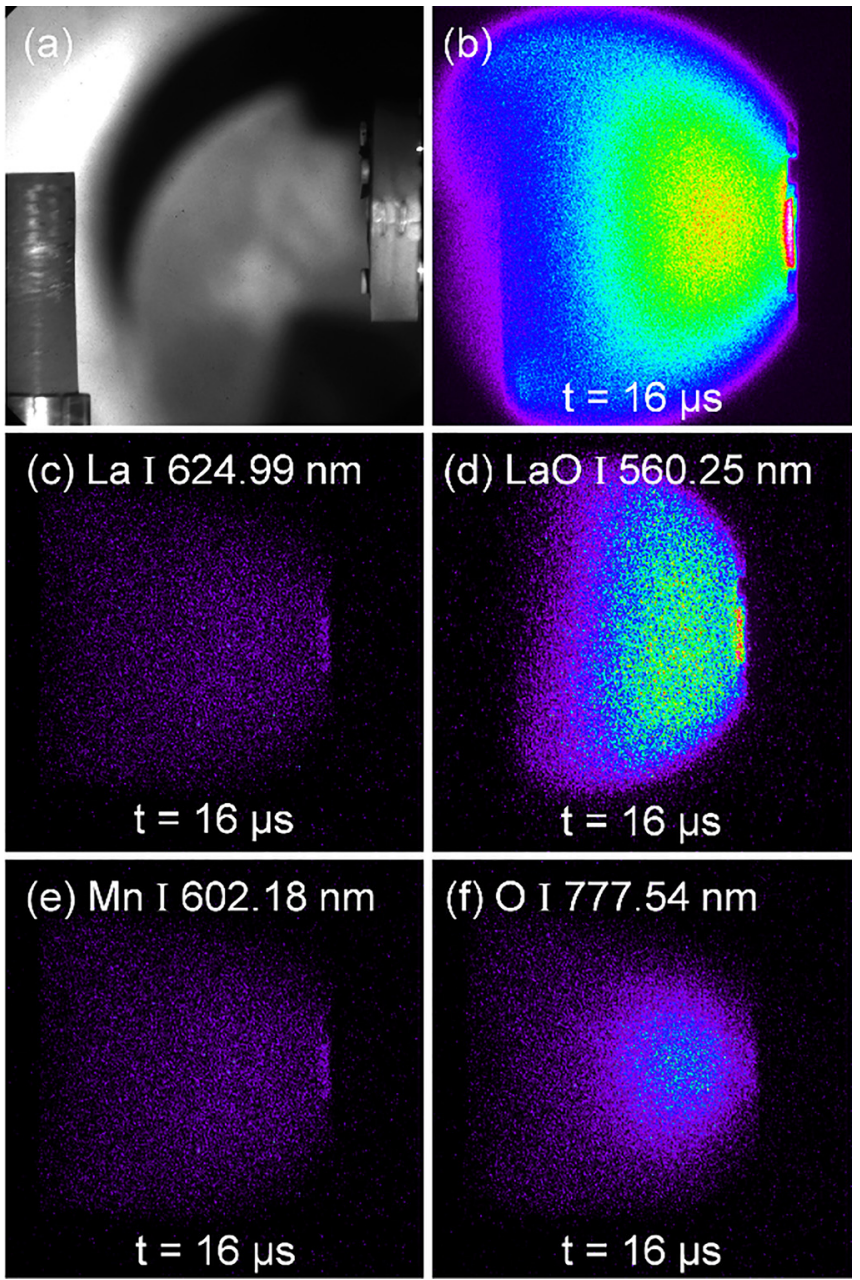

FIG. 7. ICCD images of $\mathrm{La}_{0.4} \mathrm{Ca}_{0.6} \mathrm{MnO}_{3}$ at $1 \times 10^{-1} \mathrm{mbar}_{2}$ at $t=16 \mu \mathrm{s}$. (a) Reference image with the rod target (left) and the substrate holder (right). (b) Image of visible plasma emission (300-1000 nm) showing the high emitting area close to the substrate. Wavelength selected images (AOTF) tracing the spatial distributions of the excited $\mathrm{La} \mathrm{I}(\mathrm{c}), \mathrm{LaO} \mathrm{I}(\mathrm{d}), \mathrm{Mn} \mathrm{I}(\mathrm{e})$, and O I(f). The intensity scale is the same for all AOTF images, although transmission efficiency is wavelength dependent and are not directly comparable.

the whole path up to the substrate. In the case of $1 \times 10^{-2}$ mbar, there is no significant difference in velocities with respect to vacuum until a distance of $25 \mathrm{~mm}$ is covered, where both $\mathrm{O}_{2}$ and $\mathrm{Ar}$ background gases begin reducing the velocity of the expanding plume. In these cases, the plume lowers its velocity up to $\sim 2800 \mathrm{~m} / \mathrm{s}$ moments before reaching the substrate, with Ar showing a higher stopping ability.

This difference is more pronounced at $1 \times 10^{-1}$ mbar. $\mathrm{Ar}$ is able to drop the initial velocity of $28000 \mathrm{~m} / \mathrm{s}$ down to $\sim 2800 \mathrm{~m} / \mathrm{s}$ at a travelled distance of $15 \mathrm{~mm}$, whereas in $\mathrm{O}_{2}$, it reaches $\sim 4200 \mathrm{~m} / \mathrm{s}$ at that same distance. From there onwards, there is an exponential reduction of velocity with interesting periodic undulations (highlighted with a line in Fig. 8 for $\mathrm{O}_{2} 1 \times 10^{-1}$ mbar). We suspect these are caused by shockwaves generated by the background gas during the expansion. They are also detected in other directions in the same time frames (i.e., vertical expansion, parallel to the rod target). This is a phenomenon commonly detected by shadowgraphy methods when ablating at high background pressures. ${ }^{19}$ Finally, the arrival velocities to the substrate are $\sim 280 \mathrm{~m} / \mathrm{s}$ for $\mathrm{Ar}$ and $\sim 1400 \mathrm{~m} / \mathrm{s}$ for $\mathrm{O}_{2}$ background gas, with 


\section{Plasma front velocity at room temperature}

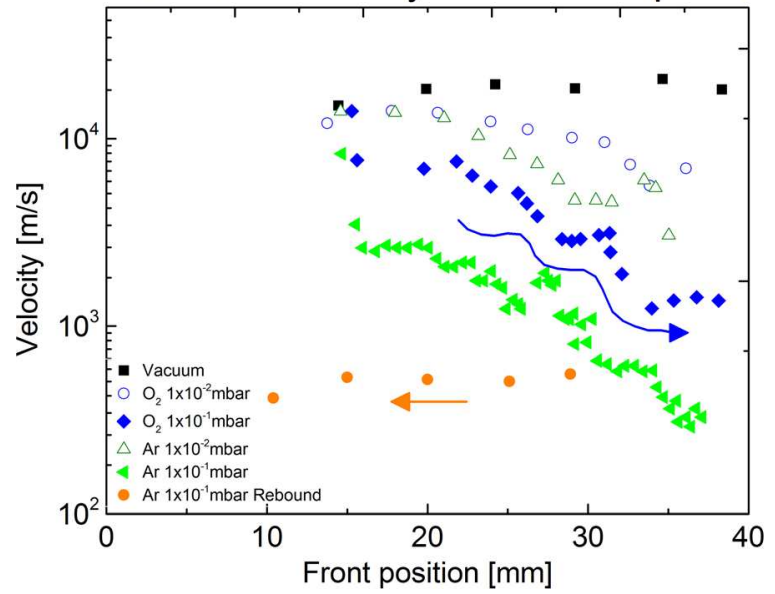

FIG. 8. Position resolved plasma velocities for different background gases and pressures at room temperature. The first $15 \mathrm{~mm}$ velocities could not be obtained due to sensor saturation (exposure gating was kept constant on purpose for all frames).

a rebound then travelling backwards at a constant speed of $\sim 530 \mathrm{~m} / \mathrm{s}$ (Ar).

\section{Resolving the initial stages of the plume expansion}

Due to the saturation of the images during the initial stages (Fig. 3 from $t=0-3 \mu \mathrm{s}$ ), the comparison between the different pressures and gases could not be performed. To capture the plasma expansion in these initial stages, the measurements were repeated using shorter gate times (10 ns vs. $50 \mathrm{~ns}$ ), which reduced the saturation of the sensor and allowed the visualization of the plume below the $3 \mu$ s timeframe. The results are plotted in Fig. 9 and confirm the dynamics observed when using $\mathrm{O}_{2}$ or Ar.

The distribution of the plume species in vacuum is forward peaked with $1 \times 10^{-2}$ mbar already changing its shape into a spherical front. The interesting events take place at the highest pressure of $1 \times 10^{-1}$ mbar where the background gas strongly opposes the free expansion of the plasma plume. It divides the plume into two regions of light emission (plume splitting) and the Ar background gas turns it into an arrow shaped emission area (with higher intensity than $\mathrm{O}_{2}$ ) as the plasma species breach the background gas (both effects have been highlighted using dashed curves in Fig. 9). Above $3 \mu \mathrm{s}$, the captured light was insufficient for imaging.

An analysis of the spatial distributions of species at $1 \times 10^{-1}$ mbar using the AOTF setup is shown in Fig. 10 . The Ag I spatial distributions are reported and also the reaction of the background gas species $\mathrm{O}_{2}$ and $\mathrm{Ar}$ when being displaced by the Ag plume.

For the $\mathrm{O}_{2}$ background, the aforementioned division of the plume in the early stages is evidently solely composed of Ag species. The O I excitation is slightly ahead and enclosing the Ag plasma. In the initial stages $(0.5 \mu \mathrm{s}-1.2 \mu \mathrm{s})$, a propagation of excitations can be seen in the $\mathrm{O}_{2}$ background, probably a shockwave excitation travelling through the background gas. Above $1.2 \mu \mathrm{s}$, the O I transforms into a hemispherical shape opposing the Ag species and, around $\sim 2.5 \mu \mathrm{s}$, forces them into such a hemispherical shape.

With the Ar background gas, the Ag I dynamics show the characteristic arrow-like shape. The Ar I shape is atypical as it is spherical already at the early stages of expansion and

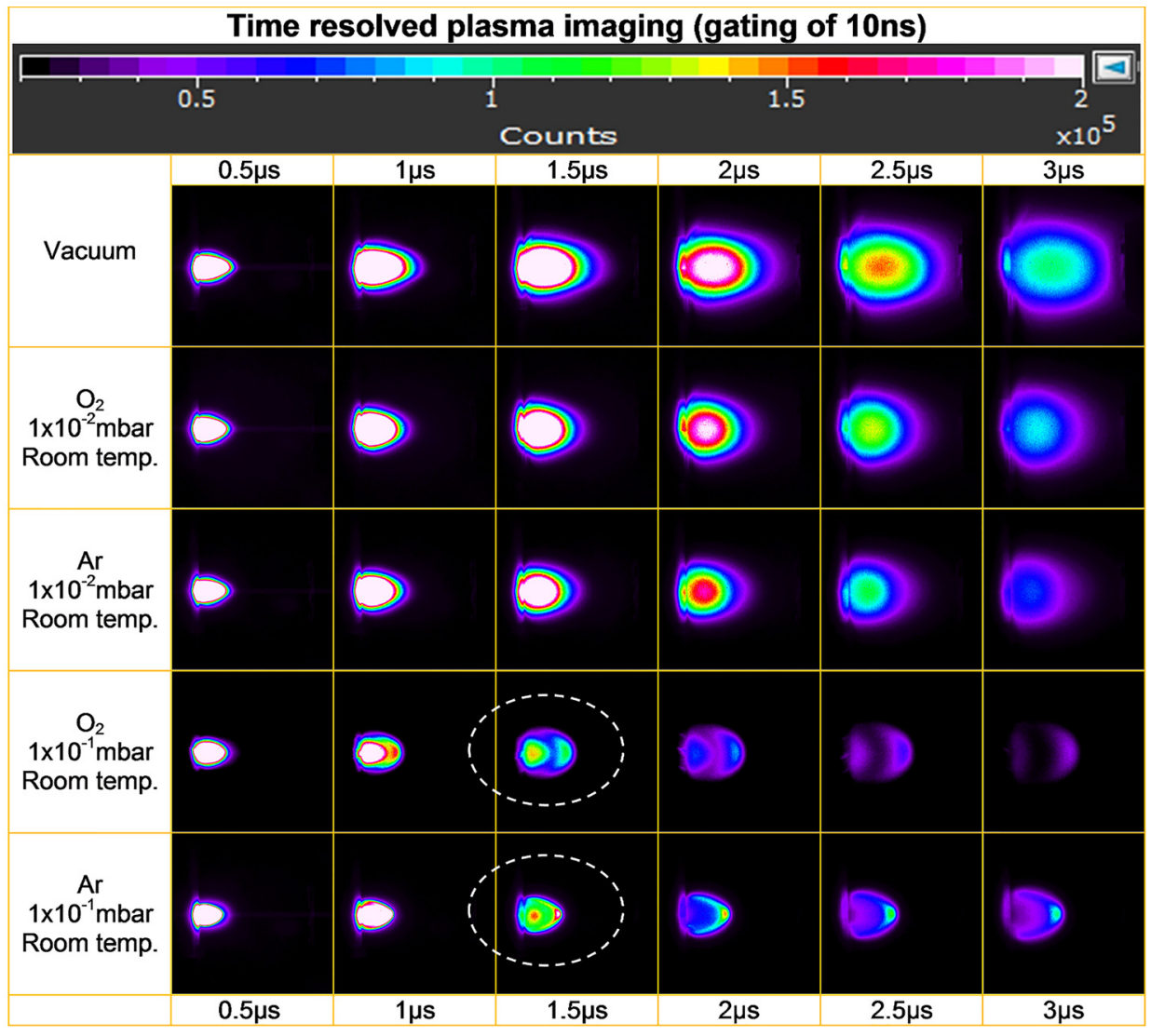

FIG. 9. Time resolved plasma imaging for vacuum, $1 \times 10^{-2}$ mbar and $1 \times 10^{-1}$ mbar for $\mathrm{O}_{2}$ and $\mathrm{Ar}$ background gases without substrate heating. The plume splitting has been highlighted using dashed curves. 


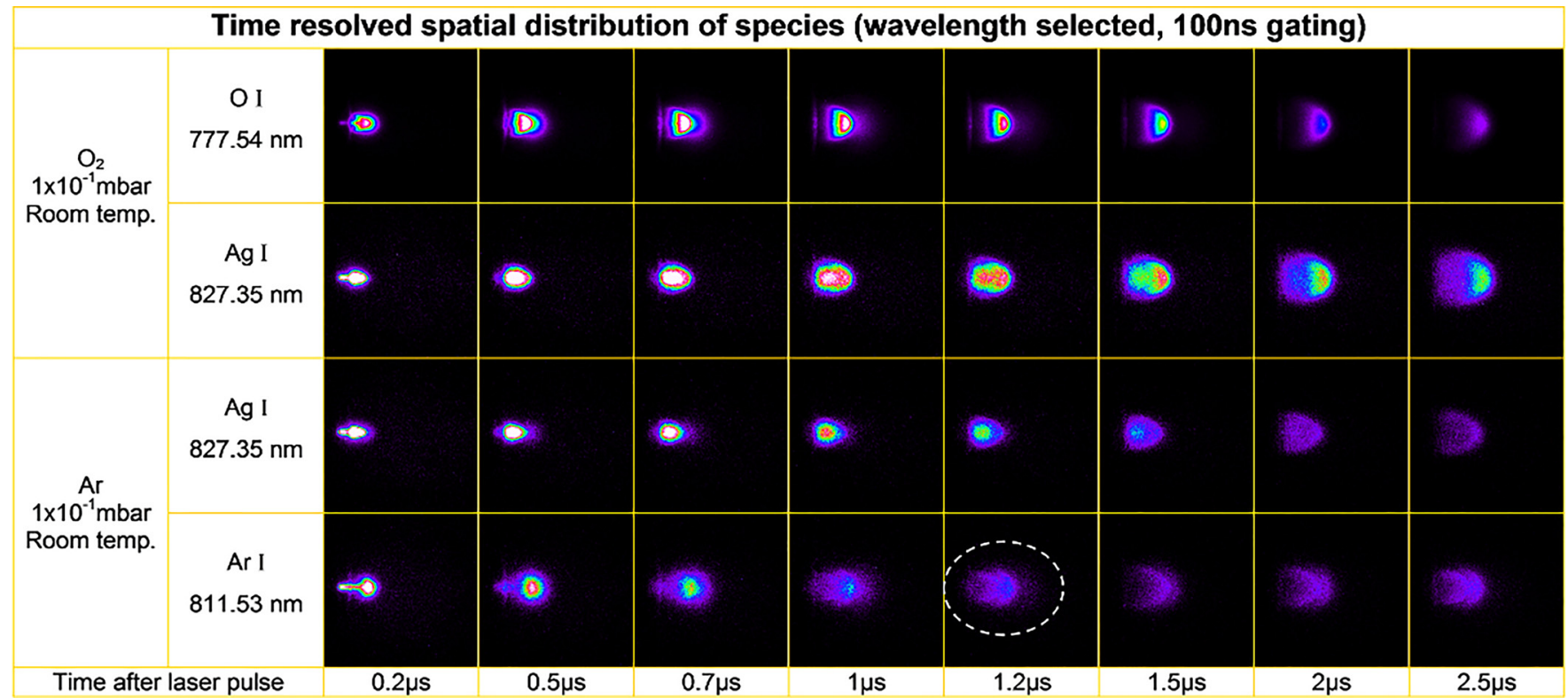

FIG. 10. Species selected imaging of the early expansion stages for $1 \times 10^{-1}$ mbar $\mathrm{O}_{2}$ and Ar. All images share the same intensity scales, although the reported intensities are dependent on wavelength choice and filter efficiency, and are not directly comparable. The re-shaping of the Ar I distribution has been highlighted using a dashed curve.

located just in front of the Ag I plume. This Ar I emitting sphere remains until $1 \mu \mathrm{s}$ when it seems to be penetrated by the energetic Ag plume, forming two complementary shapes as shown in Figs. 11(a) and 11(b) at the $1.4 \mu$ s timeframe (the repetition of the boomerang-like shape of Ar I is probably due to a shockwave excitation).

\section{B. Substrate heating influence on plasma plume expansion}

The results shown so far were for room temperature depositions, which is not necessarily the case for most PLD depositions. We now add substrate heating to the experiments while keeping all other parameters as before to understand its influence on the plasma plume expansion dynamics.

As expected, there is no effect of substrate heating $\left(600{ }^{\circ} \mathrm{C}\right)$ on the plume expansion for vacuum conditions (Fig. 12). We then focused on the $1 \times 10^{-1}$ mbar pressure cases. In both $\mathrm{O}_{2}$ and Ar background gases, the main finding is the same: higher substrate temperatures bring a much shorter time of arrival to the substrate and reduced emission intensities compared to room temperature experiments. The arrival time for $\mathrm{O}_{2}$ is now $6 \mu \mathrm{s}$ vs. previous $10 \mu \mathrm{s}$, whereas for $\mathrm{Ar}$, it is $<13 \mu \mathrm{s}$ vs. $28 \mu$ s previously. The plume front for $\mathrm{O}_{2}$ at $5 \mu$ s shows lower intensities than its lateral sides due to the $\mathrm{O}_{2}$ density decay in the proximity of the heated substrate. This was not the case at room temperature and highlights the reduction in gas density as the substrate is approached. Furthermore, the light emitting volume above the substrate shows lower intensities when compared to the room temperature case with maximums of 270000 counts per pixel without heating vs. 70000 counts per pixel for $600^{\circ}$.

In the case of Ar, the emission intensity is much lower above $5 \mu$ s than for room temperature experiments, this is, once the plume has already reached the $25 \mathrm{~mm}$ position and is only $15 \mathrm{~mm}$ away from the substrate. This suggests a lower

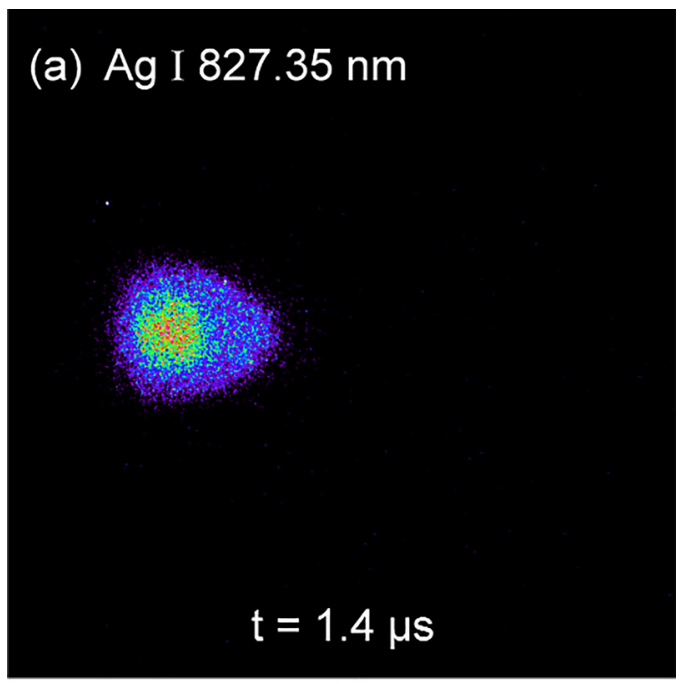

(b) $\operatorname{Ar} I 811.53 \mathrm{~nm}$

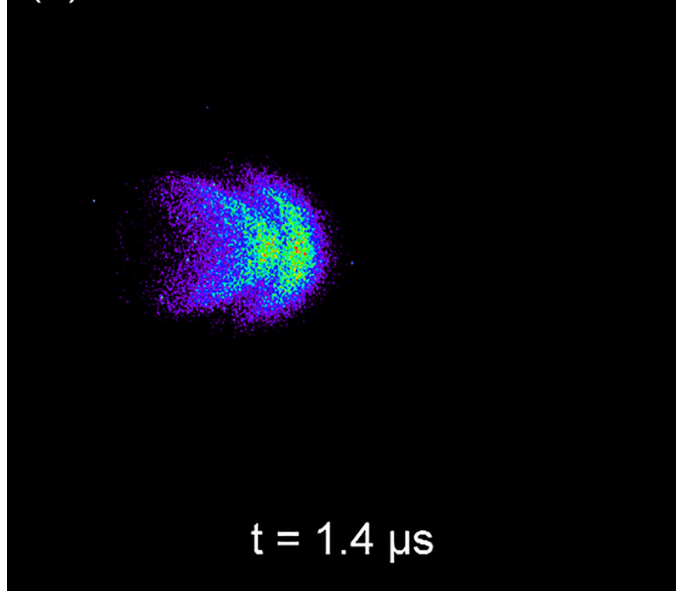

FIG. 11. Complimentary images of Ag I and Ar I spatial distributions at the same time frame of $1.4 \mu \mathrm{s}$. 100 accumulations were used to increase the image resolution. The gradients are normalized to the maximum counts for each image. 


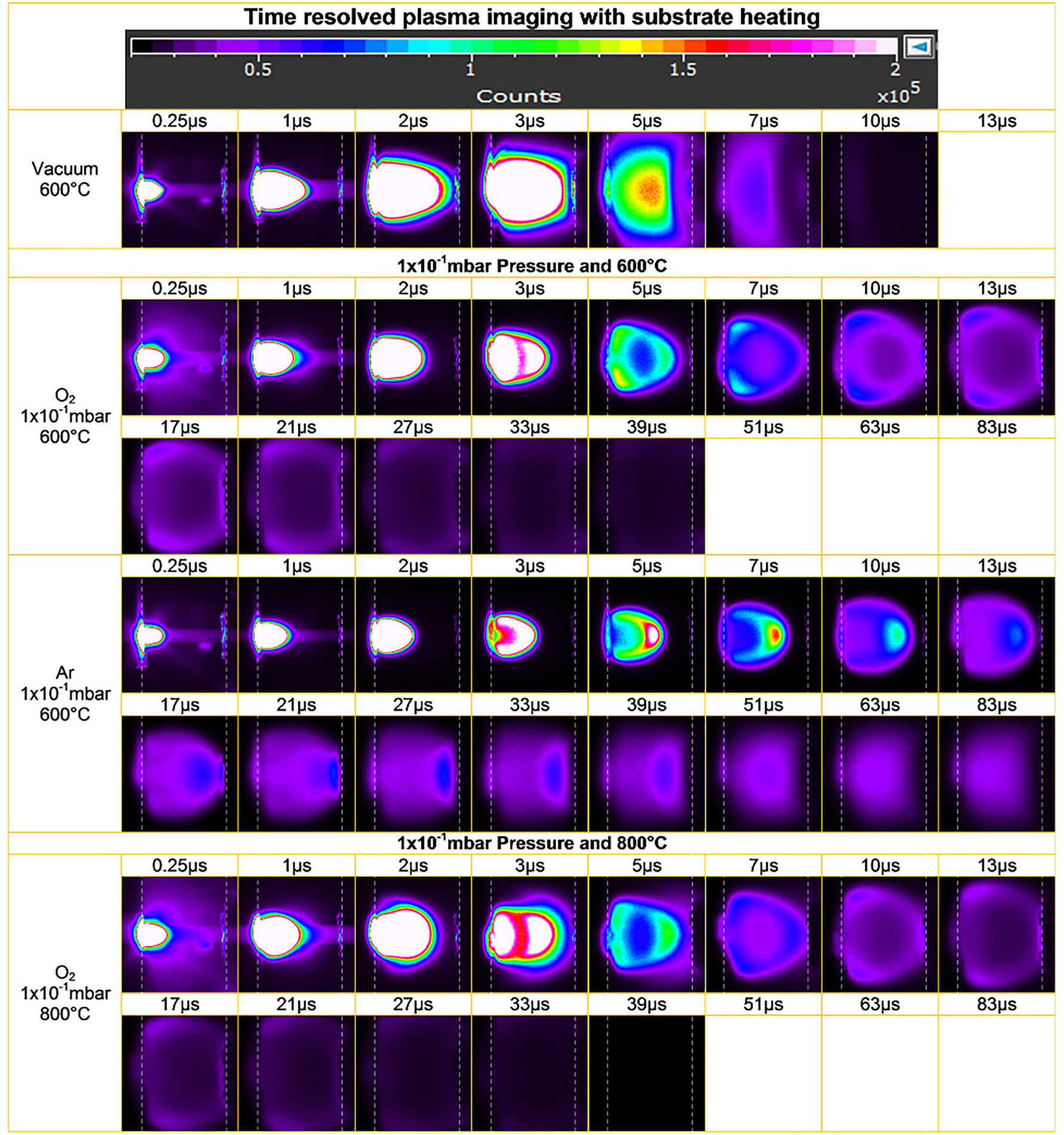

FIG. 12. Time resolved plasma imaging for vacuum, $1 \times 10^{-2} \mathrm{mbar}$ and $1 \times 10^{-1} \mathrm{mbar}$ for $\mathrm{O}_{2}$ and Ar background gases with substrate heating. The intensity scale is the same as with the RT experiments.

number of collisions and excitations when arriving at the substrate due to the reduced background gas density.

An estimation of this density gradient due to heat transfer is presented in Refs. 10 and 11 in which a conductive heat transfer, a wall temperature jump, and a linear temperature profile are assumed. However, this is not a simple task as the gas rarefaction and thus the heat transfer regime, defined by the Knudsen number $(K=\lambda / d$, where $\lambda$ is the mean free path and $d$ is the characteristic length, in this case the target to substrate distance of $40 \mathrm{~mm}$ ) is not in the conductive regime $(\mathrm{Kn} \gg 1)$, but in the Knudsen regime $(1>K n>0.01)$. In this regime, heat transfer is governed by viscosity as well as by molecular phenomena. ${ }^{20}$ Analytical equations are available for the conductive regime and the free molecular regime $(K n>1$ : heat transfer is governed by the molecular flow and hence directly proportional to the gas pressure), but not for the Knudsen regime in which numerical methods are needed. Nonetheless, it is possible to delimit 
the range of densities by resolving for the other two heat transfer regimes for which analytical equations are available.

In the viscous regime $(K n \gg 1)$, the Fourier equation $q_{x}=-k d T / d x$ with a temperature jump boundary condition is solved ${ }^{20}$ together with the energy balance equation $d q_{x} /$ $d x=0$. For this particular case of heat transfer between two parallel planes, the boundary conditions to define the constants resulting from the integration are

$$
T-T_{0}=\left\{\begin{array}{cl}
\zeta_{T} l \frac{d T}{d x} & \text { at } x=0 \\
\Delta T-\zeta_{T} l \frac{d T}{d x} & \text { at } x=d .
\end{array}\right.
$$

Obtaining the following temperature distribution:

$$
T(x)=T_{0}+\Delta T\left[\left(\frac{x}{d}+\frac{\zeta T}{\delta}\right)\left(1+\frac{2 \zeta_{T}}{\delta}\right)^{-1}\right], \quad \text { for } K n \ll 1,
$$

where $d=40 \mathrm{~mm}, \delta=\frac{1}{K n}$ is the gas rarefaction parameter, $\zeta_{T}$ is the temperature jump coefficient with a recommended value of $\zeta_{T}=1.954,{ }^{20}$ and $l$ is the Knudsen layer thickness.

In the free molecular regime $(K n \ll 1)$, the linearized Boltzmann equation is solved analytically, see chapter 4.2 in Ref. 21, giving the following temperature profile:

$$
T=T_{0}+\frac{1}{2} \Delta T, \text { for } K n \gg 1 .
$$

This means that the temperature is constant between the walls and equal to the average value. In addition, the target temperature due to radiative heat transfer is also taken into account from experimental measurements with $\sim 220^{\circ} \mathrm{C}$ $\left(210^{\circ} \mathrm{C}\right.$ at $1 \times 10^{-1}$ mbar $\mathrm{Ar}$ and $235^{\circ} \mathrm{C}$ in vacuum) and $\sim 290^{\circ} \mathrm{C}\left(280^{\circ}\right.$ at $1 \times 10^{-1} \mathrm{mbar} \mathrm{Ar}$ and $300^{\circ} \mathrm{C}$ in vacuum $)$ for the $600^{\circ} \mathrm{C}$ and $800^{\circ} \mathrm{C}$ cases, respectively.

The temperature and density profiles (ideal gas law) are shown in Figs. 13 and 14, respectively. The solution of the equations show that the heat transfer from the walls to the background gas is reduced with increasing vacuum and

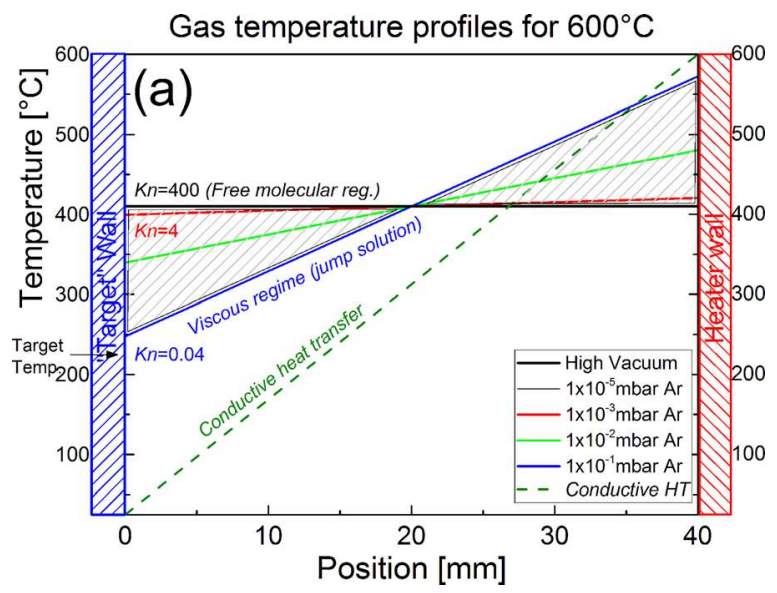

FIG. 13. Background gas temperature profiles for different pressures according to the heat transfer regime (defined by the Knudsen number) for two parallel and infinite plates at different temperatures. the discontinuity in temperature between both differing phases is seen (the temperature of the gas is not equal to that of the wall, which is emulated by the "jump" boundary solution). For the given pressures, the assumption of a conductive heat transfer regime is not ideal and gas densities are underestimated in the proximity of the heater and overestimated in the proximity of the target. This can be clearly seen in Fig. 14(a).

Nonetheless, the conclusion is similar, substrate heating has a strong influence on the background gas density which for the case of $600^{\circ} \mathrm{C}$ can be estimated as an average density of $\sim 45 \%$ RT density, while for $800^{\circ} \mathrm{C}$, it translates into $\sim 37 \%$.

In addition, the rebound effect appears, but with a diminished intensity and at a time frame of $17 \mu$ s instead of the $31 \mu \mathrm{s}$ (RT). Interestingly in the heated case, there is no non-light-emitting volume and the rebound can be seen starting from the substrate surface (Fig. 15). This is a direct consequence of the reduced gas density in the proximity of the substrate holder due to its heating, which causes less gas species to be trapped although the overall chamber pressure remains the same as before. In terms of rebound velocity in
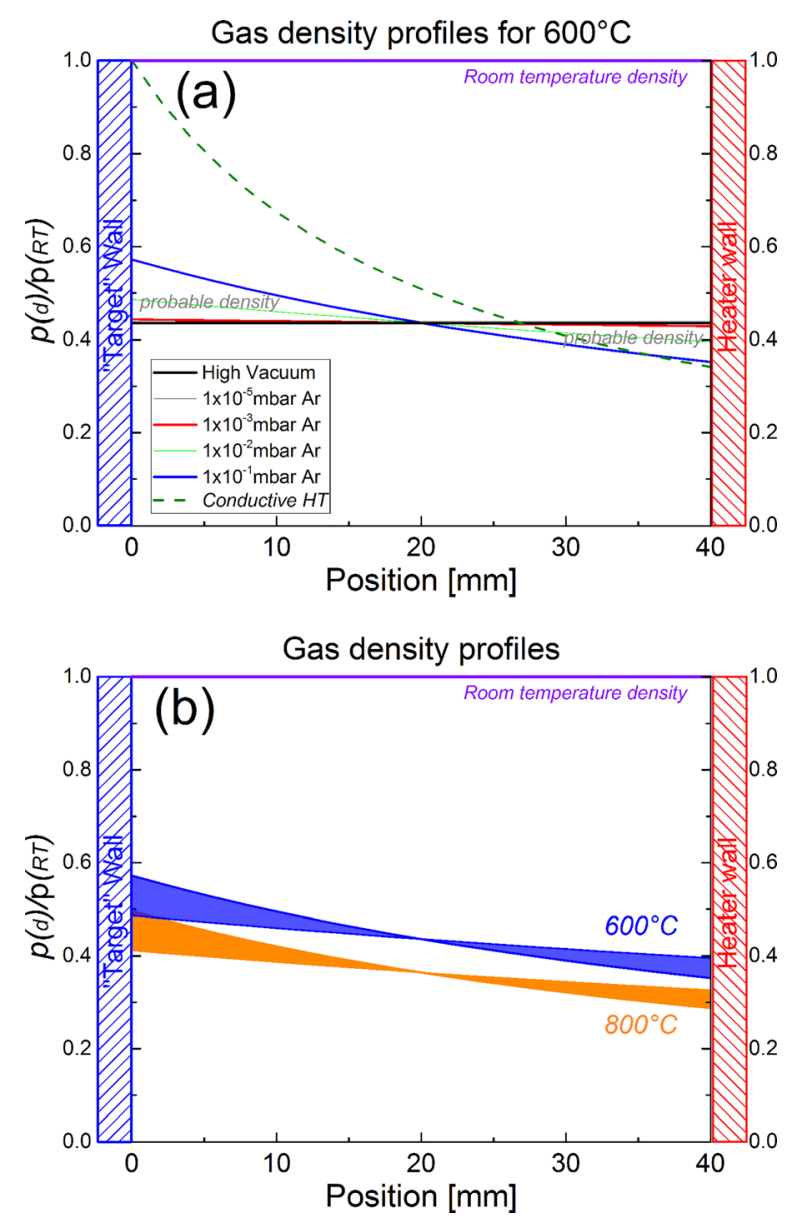

FIG. 14. Background gas density profiles for different pressures according to the heat transfer regime (defined by the Knudsen number) for two parallel and infinite plates at different temperatures (a). Probable gas density profiles referenced to room temperature density for $600^{\circ} \mathrm{C}$ and $800^{\circ} \mathrm{C}$ substrate temperature (b). Note: the target wall has different temperatures according to experimental measurements during heating $\left(\sim 100^{\circ} \mathrm{C}\right.$ and $\sim 150^{\circ} \mathrm{C}$ for the $600{ }^{\circ} \mathrm{C}$ and $800^{\circ} \mathrm{C}$ cases respectively). 

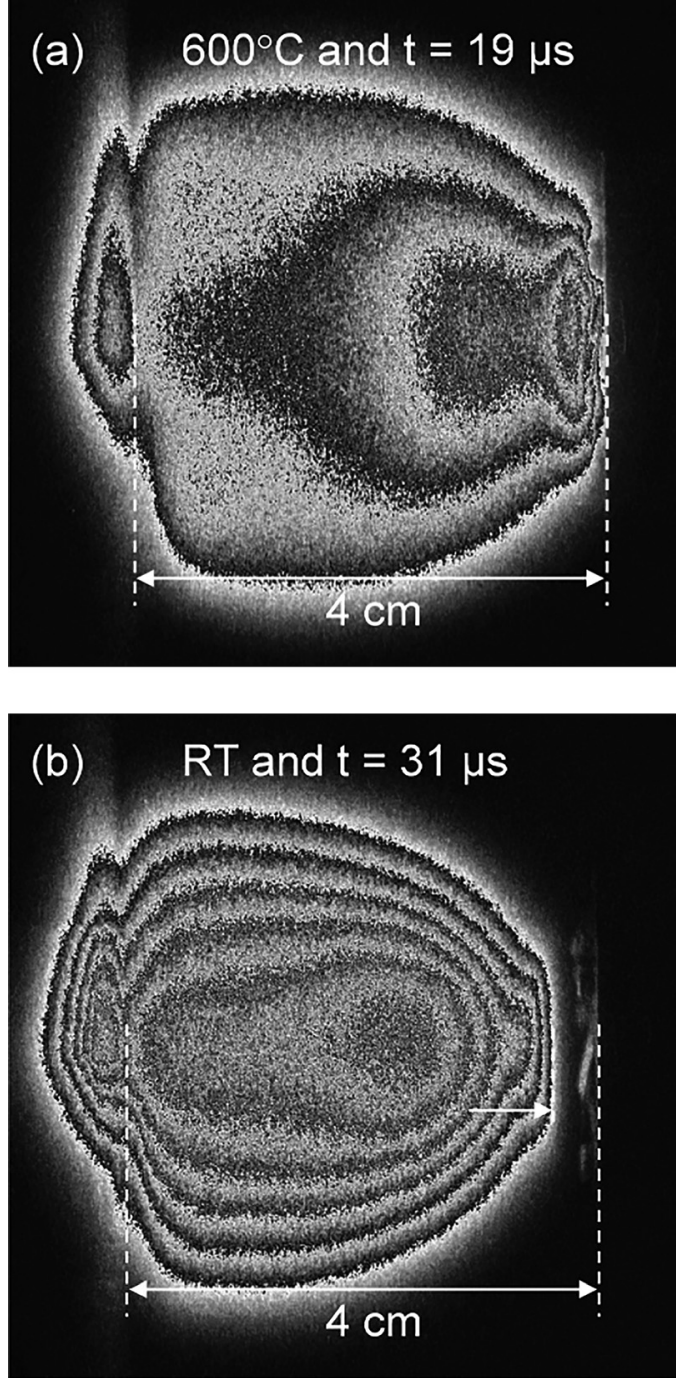

FIG. 15. Rebound effect in Ar at $1 \times 10^{-1}$ mbar for substrate heating at $600^{\circ}$ (a) and at room temperature (b). The position of the target and the substrate is highlighted to show the non-emitting volume in the room temperature case (b).

Ar, contrary to the room temperature case, for $600^{\circ} \mathrm{C}$, there is no increase in velocity. The plasma emitting species arrive at the substrate with a velocity of $\sim 1400 \mathrm{~m} / \mathrm{s}$ and rebound with a velocity of $\sim 830 \mathrm{~m} / \mathrm{s}$.

\section{Analysis of position-resolved plasma velocities}

The position-resolved velocity charts provide a similar picture and are shown in Fig. 16. The plasma expansion in vacuum is not influenced by the substrate heating and retains a quasi-constant velocity of $\sim 20000 \mathrm{~m} / \mathrm{s} . \mathrm{O}_{2}$ at $1 \times 10^{-1}$ mbar (Fig. 16(a)) shows very similar velocity values for heated and unheated substrate during the initial $25 \mathrm{~mm}$ after which $600{ }^{\circ} \mathrm{C}$ and $800^{\circ} \mathrm{C}$ temperatures maintain velocities of $4000-5500 \mathrm{~m} / \mathrm{s}$ (with $800^{\circ} \mathrm{C}$ slightly faster than the $600{ }^{\circ} \mathrm{C}$ case) while the unheated expansion reduces its velocity considerably in the last $15 \mathrm{~mm}$ (down to $1500 \mathrm{~m} / \mathrm{s}$ ). This is no surprise as the changes in gas density are expected to increase the closer one gets to the heated area (with diminishing density for the heated cases). Additionally, the
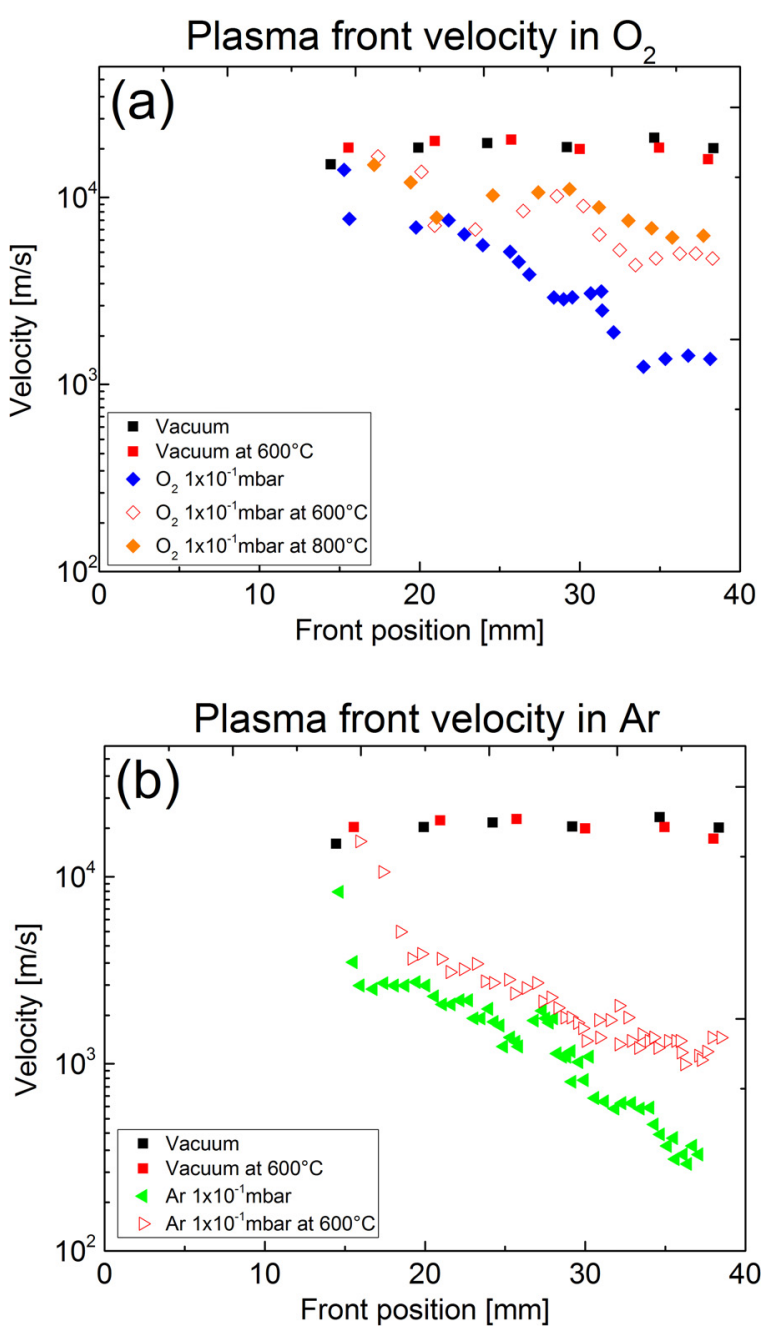

FIG. 16. Times of arrival to substrate for different background gases, pressures, and substrate temperatures, (a) for room temperature measurements and (b) for high temperature ones.

undulation of the velocity values is also present in the heated cases.

With respect to $\mathrm{Ar}$ (Fig. 16(b)), the results are similar but with a stronger reduction of velocities. The plasma expansion when heating the substrate to $600{ }^{\circ} \mathrm{C}$ diverges from the nonheated one already at $20 \mathrm{~mm}$ distance and keeps a higher velocity in the last $5 \mathrm{~mm}(1400 \mathrm{~m} / \mathrm{s}$ vs. $280 \mathrm{~m} / \mathrm{s})$.

\section{Times of arrival}

Although the velocity charts have been shown previously, it is also relevant to report on the time of arrival at the substrate for the different pressures and heating temperatures, as there is a marked velocity variability throughout the entire expansion (except for vacuum).

The results are reported in Fig. 17 in which in vacuum the plasma species arrive at the substrate in the shortest time $(\sim 1.75 \mu \mathrm{s})$. At $1 \times 10^{-2}$ mbar, both $\mathrm{O}_{2}$ and Ar background gases are able to affect the expansion, with arrival times of $2.75 \mu \mathrm{s}$ and $3 \mu \mathrm{s}$, respectively.

At the highest pressure of $1 \times 10^{-1}$ mbar, the arrival times when using $\mathrm{O}_{2}$ or $\mathrm{Ar}$ are considerably different, with the species expanding in $\mathrm{O}_{2}$ needing $\sim 9.75 \mu$ s to reach the 


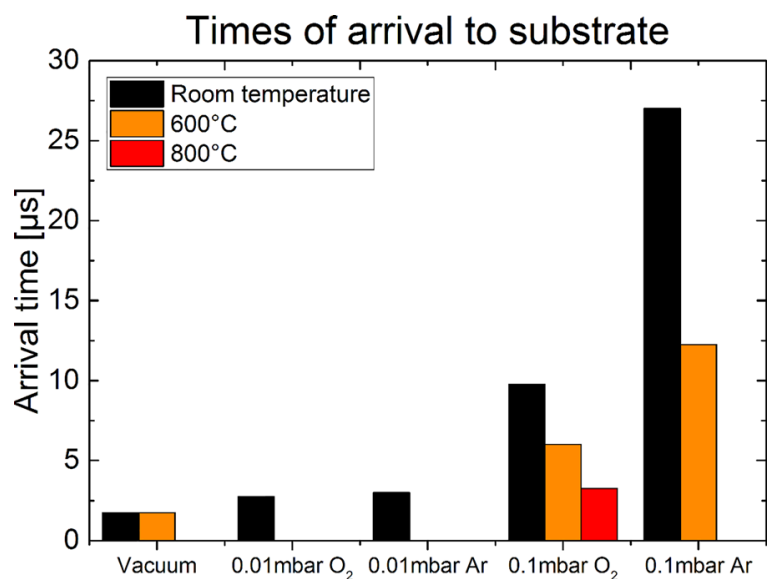

FIG. 17. Times of arrival to substrate for different background gases, pressures, and substrate temperatures.

substrate (at room temperature), whereas for those expanding in Ar needing $28 \mu$ s (three times more), both of which are in strong contrast with the arrival times in vacuum.

The aforementioned timing results are for room temperature experiments. Raising the substrate temperature causes the mentioned reduction in gas density close to the substrate. The expansion in vacuum is not affected, whereas for the experiments at $1 \times 10^{-1} \mathrm{mbar}$, the reduction in time of arrival is considerable for both $\mathrm{O}_{2}\left(t_{R T}=9.75 \mu \mathrm{s}, t_{600^{\circ}}=6 \mu \mathrm{s}\right.$, and $\left.t_{800^{\circ}}=3.25 \mu \mathrm{s}\right)$ and $\operatorname{Ar}\left(t_{R T}=27 \mu \mathrm{s}\right.$ and $\left.t_{600^{\circ}}=12.25 \mu \mathrm{s}\right)$. For $\mathrm{Ar}$ at $600{ }^{\circ} \mathrm{C}$, it is $\sim 45 \%$ for the time of arrival at $\mathrm{RT}$, whereas for $\mathrm{O}_{2}$, it is $\sim 61 \%$ and $\sim 33 \%$ at $600^{\circ} \mathrm{C}$ and $800^{\circ} \mathrm{C}$, respectively. These experimental values are in agreement with the estimated gas densities due to heating from section B: $\sim 45 \%$ at $600{ }^{\circ} \mathrm{C}$ and $\sim 37 \%$ at $800^{\circ} \mathrm{C}$.

\section{CONCLUSIONS}

Time resolved imaging of the plasma expansion for the different deposition pressures and temperatures provides a detailed insight into the PLD process. In vacuum conditions, the expansion is strongly forward peaked and the high arrival velocities of the species to the substrate cause them to rebound. Once the pressure is sufficient, there is a transition from a forward peaked deposition to a spherical one. However, during the initial stages of the expansion, the plume is not affected by the surrounding background gas. For the same given pressure, Ar shows a higher stopping ability than molecular $\mathrm{O}_{2}$ (related to its higher mass and the van der Waals volume).

At the highest tested pressure of $1 \times 10^{-1}$ mbar, both background gases show that upon arrival of the species to the substrate a transient locally rich gas environment is created above the substrate ( $\sim 5 \mathrm{~mm}$ thickness). We believe that this is a consequence of the trapping and compression of background gas species between the expanding plume and the substrate holder. In the case of Ar, an unexpected rebound of plasma species is detected rebounding all the way back to the originally ablated target. Surprisingly, the rebound velocity is higher than the arrival one, evidencing a spring-like behaviour. This rebound follows a different mechanism than the one in vacuum, as it is not caused by a direct reflection from the substrate surface. In the case of $\mathrm{O}_{2}$, an area of intense emission of light is seen which surpasses the intensities measured at the plasma plume front at 3-5 $\mu \mathrm{s}$. This emission is mostly generated by $\mathrm{Ag} \mathrm{I}$ and a metal oxygen species (evidenced for $\mathrm{LaO}$ ). A slight rebound is also seen for $\mathrm{O}_{2}$, but is masked by the higher intensities in the near substrate region.

The effect on the plasma dynamics due to substrate heating was also analysed. It shows no influence for vacuum conditions, and for higher pressures, the results evidence a gradual decay in background gas density the closer one gets to the heated substrate. Expansion velocities are increased and consequently times of arrival are reduced. The $\mathrm{O}_{2}$ rich layer and the rebound effect continue to appear, but with reduced intensity. Analytical estimations of the gas densities were shown that would allow adjusting the background gas pressure to achieve similar density if the substrate temperature is varied.

\section{ACKNOWLEDGMENTS}

Financial supports from the Paul Scherrer Institut and the Swiss National Science Foundation (SNF/Project No. 00021_143665) are gratefully acknowledged.

${ }^{1}$ T. Venkatesan, J. Phys. D: Appl. Phys. 47, 034001 (2014).

${ }^{2}$ D. B. Chrisey and G. K. Hubler, Pulsed Laser Deposition of Thin Films (Wiley, New York, 1994), Vol. 1.

${ }^{3}$ R. Eason, Pulsed Laser Deposition of Thin Films: Applications-LED Growth of Functional Materials (John Wiley \& Sons, 2007).

${ }^{4}$ H. M. Christen and G. Eres, J. Phys.: Condens. Matter 20, 264005 (2008).

${ }^{5}$ D. Dijkkamp, T. Venkatesan, X. Wu, S. Shaheen, N. Jisrawi, Y. Min-Lee, W. McLean, and M. Croft, Appl. Phys. Lett. 51, 619 (1987).

${ }^{6} \mathrm{~J}$. Schou, Appl. Surf. Sci. 255, 5191 (2009).

${ }^{7}$ A. Voevodin, J. Jones, and J. Zabinski, J. Appl. Phys. 88, 1088 (2000).

${ }^{8}$ D. B. Geohegan and A. A. Puretzky, "Species-resolved imaging and gated photon counting spectroscopy of laser ablation plume dynamics during KrFand ArF-laser PLD of amorphous diamond films," MRS Proc. 397, 55 (1995).

${ }^{9}$ S. Canulescu, E. L. Papadopoulou, D. Anglos, T. Lippert, C. W. Schneider, and A. Wokaun, J. Appl. Phys. 105, 063107 (2009).

${ }^{10} \mathrm{~S}$. Amoruso and R. Bruzzese, "Substrate heating influence on plume propagation during pulsed laser deposition of complex oxides," Appl. Phys. Lett. 91, 151501 (2007).

${ }^{11}$ A. Sambri, S. Amoruso, X. Wang, F. M. Granozio, and R. Bruzzese, J. Appl. Phys. 104, 053304 (2008).

${ }^{12}$ S. Amoruso, C. Aruta, R. Bruzzese, X. Wang, and U. Scotti di Uccio, Appl. Phys. Lett. 98, 101501 (2011).

${ }^{13}$ M. R. Predteceensky and A. P. Mayorov, Appl. Supercond. 1, 2011 (1993).

${ }^{14}$ W. M. Haynes, CRC Handbook of Chemistry and Physics (CRC Press, 2014).

${ }^{15}$ F. Phelps III, MIT Wavelength Tables, Wavelengths by Element (MIT Press, Cambridge, MA, 1982), p. 810.

${ }^{16}$ S. S. Batsanov, Inorg. Mater. 37, 871 (2001).

${ }^{17}$ S. I. Anisimov, B. S. Luk'yanchuk, and A. Luches, Appl. Surf. Sci. 96-98, 24 (1996).

${ }^{18}$ D. Marla, U. V. Bhandarkar, and S. S. Joshi, J. Appl. Phys. 109, 021101 (2011).

${ }^{19}$ S. Heiroth, J. Koch, T. Lippert, A. Wokaun, D. Günther, F. Garrelie, and M. Guillermin, J. Appl. Phys. 107, 014908 (2010).

${ }^{20} \mathrm{~K}$. Jousten, Handbook of Vacuum Technology (John Wiley \& Sons, 2008).

${ }^{21}$ M. Kogan, Rarefied Gas Dynamics (Plenum Press, New York, 1969), Vol. 2, p. 1 . 